\title{
The System for Observing Fitness Instruction Time (SOFIT) as a Measure of Energy Expenditure During Classroom-Based Physical Activity
}

\author{
Jeffery J. Honas, Richard A. Washburn, Bryan K. Smith, \\ Jerry L. Greene, Galen Cook-Wiens, and Joseph E. Donnelly
}

\begin{abstract}
The aim of this investigation was to develop an equation to estimate physical activity energy expenditure (PAEE) during a 10-min physically active academic lesson using The System for Observing Fitness Instruction Time (SOFIT) and demographic information. PAEE (portable indirect calorimeter) and physical activity (SOFIT) were simultaneously assessed in 38, 2nd through 5th grade children. PAEE and SOFIT were $3.04 \pm 1.1(\mathrm{kcal} / \mathrm{min})$ and $3.8 \pm 0.4$ (score), respectively. PAEE was predicted from SOFIT score and body weight $[\mathrm{PAEE}(\mathrm{kcal} / \mathrm{min})=(1.384 * \mathrm{SOFIT}+0.084 *$ weight $(\mathrm{kg})-5.126), R=.81, \mathrm{SEE}=1.23 \mathrm{kcal} / \mathrm{min}]$. PAEE measured by indirect calorimeter and predicted from SOFIT and body weight were $3.04 \pm 1.1(\mathrm{kcal} / \mathrm{min})$ and $3.04 \pm 0.9$ $\mathrm{kcal} / \mathrm{min}$ ) respectively. SOFIT and body weight may provide a useful measure of PAEE associated with classroom based physical activity.
\end{abstract}

The 2003-2004 National Health and Nutrition Examination Survey reported $37.2 \%$ of children ages $6-11$ were at risk for overweight ( $\geq 85$ th BMI percentile) or overweight ( $\geq 95$ th BMI percentile) compared with $29.8 \%$ observed in the 1999-2000 sample (13). Decreased physical activity energy expenditure (PAEE) is likely a major contributor to the current and increasing level of obesity in children $(3,5)$

Children spend much of their time at school and the school environment represents a suitable setting to increase PAEE $(1,12,15,17)$. Unfortunately, increased pressure placed on schools to improve academic achievement has decreased the time available for physical education classes, thus diminishing the level of physical activity during the school day. However, interventions designed to increase PAEE in the classroom by incorporating physical activity with academic lessons may have the potential to increase PAEE to levels sufficient to reduce the development of overweight and obesity, while not diminishing classroom instruction time (4).

Honas, Washburn, Smith, Greene, and Donnelly are with the Center for Physical Activity and Weight Management, University of Kansas, Lawrence, KS 66045. Cook-Wiens is with the Kansas University Medical Center, Kansas City, KS 66160. 
To assess the efficacy of classroom-based physical activity interventions to impact overweight and obesity in children, easily administered, valid, and reliable methods for assessing PAEE during classroom physical activity are needed. The System for Observing Fitness Time (SOFIT; 10) is a observation technique designed to assess physical activity levels during physical education classes and has been validated using other visual observation methods $(6,8)$, heart rate monitors $(10,14)$, pedometers (16) and accelerometers (9). The SOFIT also has been used in large physical activity studies such as the Child and Adolescent Trial for Cardiovascular Health (CATCH) (7) and Sports, Play, and Active Recreation for Kids (SPARK) (15). However, to date no studies have evaluated the validity of the SOFIT as a measure of PAEE during classroom based physical activity. The goal of the current study was to evaluate the potential of the SOFIT to estimate PAEE during physically active classroom lessons with portable indirect calorimeter as the criterion measure.

\section{Methods}

\section{Intervention}

The Physical Activity Across the Curriculum (PAAC) intervention was designed to determine if three years of increased classroom based physical activity diminishes gains in body mass index (BMI) in elementary school children. Classroom teachers were trained during a $6 \mathrm{hr}$ in-service to use established academic curricula to deliver physically active academic lessons of moderate intensity (3-6 METS) of approximately 10 min duration, twice per day throughout the school year. For this study, we assessed 38 PAAC lesson led by 19 different classroom teachers. Examples of lessons included jumping to an "invisible" jump rope or performing calisthenics such as jumping-jacks or squats while reciting multiplication tables. Geography (e.g., North, South, East, and West) was taught by having children walk or run to the appropriate area designated for each direction. Spelling was taught by having children jump on appropriate letters placed on the floor, etc.

\section{Participants}

Participants were volunteers from classrooms in grades 2 through 5 from three suburban schools in Northeast Kansas participating in the PAAC intervention. Parental consent and student assent forms were delivered to the PAAC schools and were distributed by the classroom teachers. Signed consent and assent forms were returned from a convenience sample of 38 children. All procedures were approved by the Institutional Review Board at the University of Kansas.

\section{Assessments}

Study procedures were explained to each child and classroom teacher before the assessment. Before the PAEE assessments, age and gender were recorded. In addition, height (nearest $0.1 \mathrm{~cm}$ ) and weight (nearest $0.1 \mathrm{~kg}$ ) were assessed with participants in street clothes (shoes removed) using a mobile stadiometer (Model 
\# IP0955, Invicta Plastics Limited, Leicester, England) and a digital scale (PS-6600, Befour Inc., Saukville, WI), respectively. Body mass index (BMI) was calculated by dividing weight in kilograms by height in meters squared. PAEE was assessed during 38 PAAC lessons using a portable indirect calorimeter with simultaneous evaluation using SOFIT.

Portable Indirect Calorimetry. Energy expenditure of PAAC lessons was assessed using a Cosmed K4b ${ }^{2}$ (Cosmed, Rome, Italy; Figure 1). The Cosmed measures breath-by-breath ventilation, expired $\mathrm{O}_{2}$ and $\mathrm{CO}_{2}$ and has been validated previously (11). After a minimum 30-min warm-up, the Cosmed $\mathrm{O}_{2}$ and $\mathrm{CO}_{2}$ analyzers were calibrated with known gases. The flow turbine was calibrated using a 3.00L syringe. The participant was excused from the classroom $5 \mathrm{~min}$ before the start of the scheduled PAAC lesson to be fitted with the Cosmed. The light weight $(\sim 1.5 \mathrm{~kg})$, portable system was attached by harness around the waist and shoulders of the child (Figure 1) and was started and checked for leaks around the facemask and hoses. After the participant entered the room the teacher initiated the 10 min PAAC lesson. The data were retrieved for analysis via serial port interface and software provided with the Cosmed and was reduced to $60 \mathrm{~s}$ epochs.

SOFIT. PAAC lessons were scored using the SOFIT, a visual observation instrument previously validated as a measure of physical activity during physical education classes (10). SOFIT scores activity using a 5-point Likert scale (1 = lying

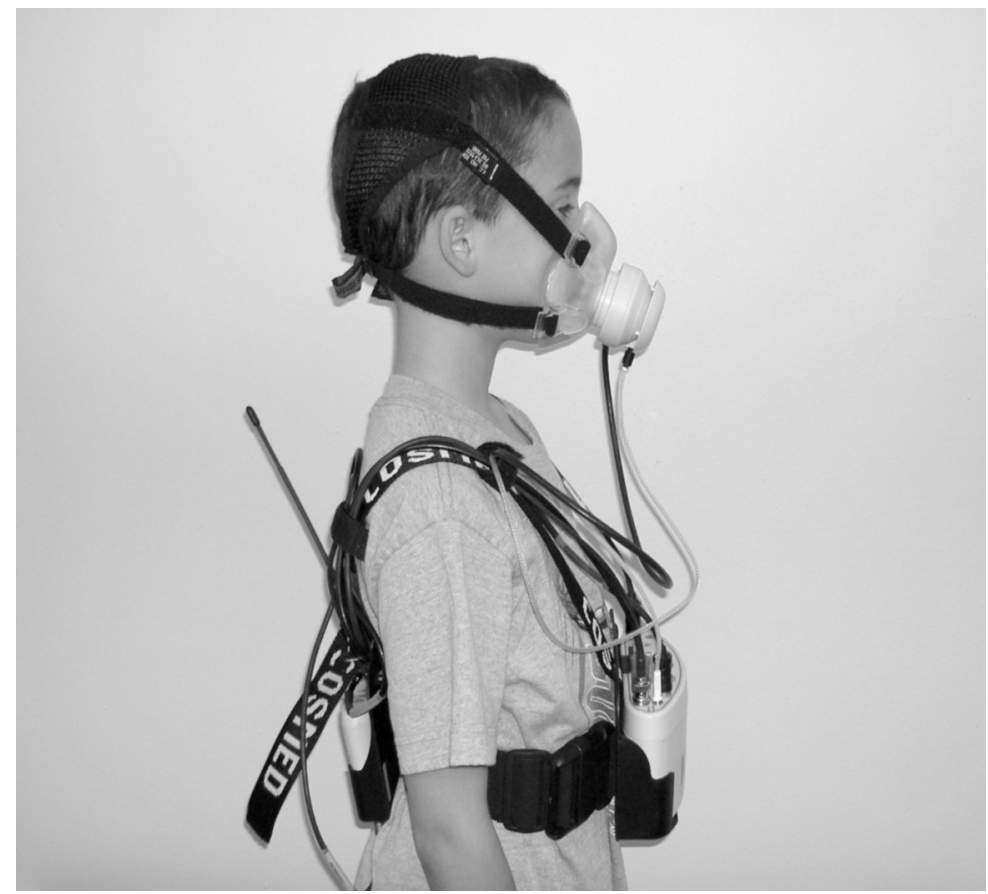

Figure 1 - Portable indirect calorimeter. 
down, $2=$ sitting, $3=$ standing, $4=$ walking, $5=$ very active $)$. The original SOFIT data collection protocol ( 3 scores each minute) was modified so that research assistants recorded physical activity level over the last $10 \mathrm{~s}$ of each 1-min interval during the PAAC lesson. Four trained research assistants participated in SOFIT scoring and demonstrated interrater reliability $\geq 0.85$.

\section{Analysis}

Descriptive statistics were calculated for the entire study population and by gender. Values are reported as mean $\pm S D$. Linear regression models were constructed with average PAEE measured by indirect calorimeter as the dependent variable, and age, gender, and body weight as independent variables. A stepwise approach was used to determine which variables, in addition to averaged SOFIT score (average of ten, 1-min values) contributed to the model based on the adjusted $\mathrm{R}$ squared statistic. An alpha level of 0.05 was used for statistical significance. All analyses were carried out using SAS (version 9.1.; SAS Institute, Cary, NC).

\section{Results}

Thirty-eight (19 female, 19 male, 34 White, 4 Hispanic) second through fifth grade students (grade 2: $n=13$, grade 3: $n=7$, grade 4: $n=11$, grade 5: $n=7$ ) were evaluated. Participant descriptive characteristics by gender are presented in Table 1. There were no significant gender differences for age, body weight, BMI, or percent overweight or at risk for overweight.

The data for SOFIT (score) and PAEE ( $\mathrm{kcal} / \mathrm{min}$ ) assessed by portable indirect calorimeter and predicted by linear regression model including SOFIT score and body weight $(\mathrm{kg})$ are presented in Table 2 . No significant gender differences were noted for either SOFIT (score) or PAEE ( $\mathrm{kcal} / \mathrm{min}$ ) assessed by indirect calorimeter. PAEE during PAAC activities was predicted from the average SOFIT score and body weight [PAEE $(\mathrm{kcal} / \mathrm{min})=1.384 *$ SOFIT $+0.084 *$ weight $(\mathrm{kg})-5.126), R=.81, \mathrm{SEE}=1.23 \mathrm{Kcal} / \mathrm{min}]$. The adjusted $\mathrm{R}$ squared is marginally improved when either age or gender are added to this model (models 5 and 6 in Table 3), however, the effects of age or gender were not statistically significant. The model with SOFIT score and weight (model 2) is recommended because it

\section{Table 1 Descriptive Characteristics of the Participants}

\begin{tabular}{lccccc}
\hline & \multicolumn{2}{c}{$\begin{array}{c}\text { Age } \\
\text { (years) }\end{array}$} & Body Wt (kg) BMI (kg/m2) & $\begin{array}{c}\text { Percent overweight or at } \\
\text { risk for overweight * }\end{array}$ \\
\hline Female & 19 & $9.3 \pm 1.3$ & $34.4 \pm 7.8$ & $18.7 \pm 3.5$ & 31.6 \\
Male & 19 & $8.9 \pm 1.4$ & $33.8 \pm 8.4$ & $18.7 \pm 3.3$ & 36.8 \\
\hline Total & 38 & $9.1 \pm 1.3$ & $34.1 \pm 8.0$ & $18.7 \pm 3.3$ & 34.2 \\
\hline
\end{tabular}

Note. BMI = Body Max Index; Values are means $\pm S D$; No significant gender differences between any variable

* $\geq 85$ th BMI percentiles from the Center for Disease Control and Prevention (2) 
Table 2 SOFIT Score, Measured and Predicted Physical Activity Energy Expenditure (PAEE)

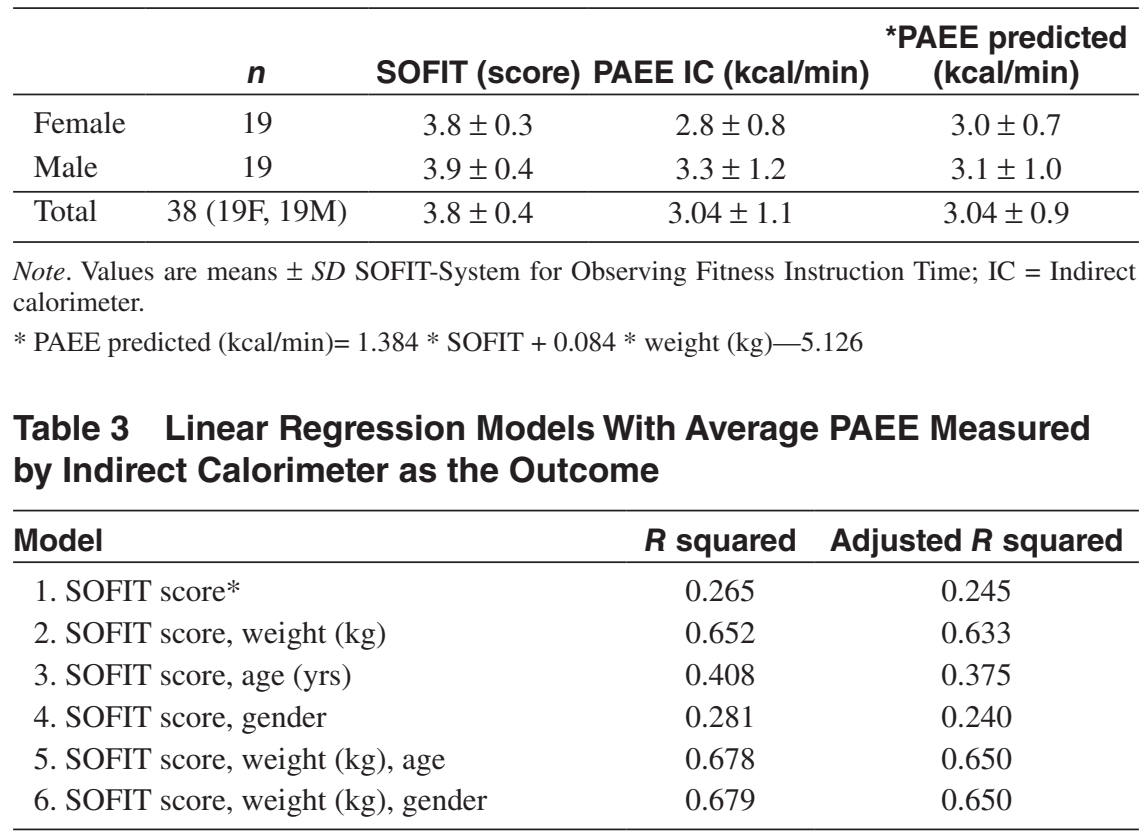

* Average over 10, 1-min observations

has similar model fit to models with three variables but is more parsimonious. PAEE measured by indirect calorimeter and predicted from SOFIT and body weight were $3.04 \pm 1.1(\mathrm{kcal} / \mathrm{min})$ and $3.04 \pm 0.9 \mathrm{kcal} / \mathrm{min})$, respectively.

\section{Discussion}

To assess the efficacy of classroom based physical activity interventions to decrease the prevalence of overweight and obesity in children, it is essential to have a valid estimate of the level of energy expenditure provided by the prescribed physical activity program. We have developed a regression equation using SOFIT score and body weight that may provide a valid estimate of energy expenditure during classroom based physical activity in elementary age boys and girls. These results are in agreement with previous studies which have shown SOFIT provides valid estimates of physical activity intensity in comparison with other objective assessment methods during physical education classes $(6,9,14,16)$.

This study evaluated the ability of SOFIT to estimate classroom based PAEE in elementary school children using energy expenditure measured by portable indirect calorimeter as a criterion measure. SOFIT offers an inexpensive, easily administered method for the estimation of PAEE suitable for use in large-scale intervention trials of increased classroom based physical activity. 
Potential study limitations include the small sample size and use of a modified version of SOFIT. During each 10 min PAAC lesson, the research assistant observed PAAC activities and recorded SOFIT (score) during the last $10 \mathrm{~s}$ of each 1-min interval. The modified SOFIT protocol used differs from the original in which 3 SOFIT scores per minute were recorded. The modified protocol has merit given the relatively consistent activity patterns within each of the 10 min physically active lesson as evidenced by limited varation in energy expenditure assessed by indirect calorimetery. In addition, the PAEE during PAAC activities was predicted from the average SOFIT scores and body weight of 38 children and were derived by evaluating several different PAAC activities delivered by 19 different classroom teachers. We are therefore unable to assess the reliability of our energy expenditure estimates either within or between teacher and lesson.

In summary, results of the current study suggest that a regression equation using a SOFIT score (modified protocol) and body weight provides a valid estimate of PAEE during classroom based physical activity in elementary school boys and girls. Further evaluation of the validity and reliability of this equation in larger samples and over a variety of classroom based physical activities is warranted.

\section{References}

1. Caballero, B., T. Clay, S.M. Davis, et al. Pathways: a school-based, randomized controlled trial for the prevention of obesity in American Indian schoolchildren. Am. J. Clin. Nutr. 78:1030-1038, 2003.

2. Center for Disease Control and Prevention Web site [Internet]. Atlanta (GA): 2000 Center for Disease Control Growth Charts; [cited 2007 June 1] www.cdc.gov/growthcharts/

3. Dencker, M., O. Thorsson, M.K. Karlsson, et al. Daily physical activity related to body fat in children aged 8-11 years. J. Pediatr. 149:38-42, 2006.

4. Dubose, K.D., M.S. Mayo, C.A. Gibson, et al. Physical activity across the curriculum (PAAC): Rationale and design. Contemp. Clin. Trials. 29(1):83-93, 2008.

5. Goldfield, G.S., R. Mallory, T. Parker, et al. Effects of open-loop feedback on physical activity and television viewing in overweight and obese children: a randomized, controlled trial. Pediatrics. 118:e157-e166, 2006.

6. Heath, E.M., K.J. Coleman, T.L. Lensegrav, and J.A. Fallon. Using momentary time sampling to estimate minutes of physical activity in physical education: validation of scores for the system for observing fitness instruction time. Res. Q. Exerc. Sport. 77:142-146, 2006.

7. Luepker, R.V., C.L. Perry, S.M. McKinlay, et al. Outcomes of a field trial to improve children's dietary patterns and physical activity. The Child and Adolescent Trial for Cardiovascular Health. CATCH collaborative group. JAMA. 275:768-776, 1996.

8. McKenzie, T.L. Observational measures of children's physical activity. J. Sch. Health. 61:224-227, 1991.

9. McKenzie, T.L., J.F. Sallis, and C.A. Armstrong. Association between direct observation and accelerometer measures of children's physical activity during physical education and recess. Med. Sci. Sports Exerc. 26:143, 1994.

10. McKenzie, T.L., J.F. Sallis, and P.R. Nader. SOFIT: System for observing fitness instruction time. J Teach Phys Educ. 11:195-205, 1991.

11. McLaughlin, J.E., G.A. King, E.T. Howley, D.R. Basset, Jr., and B.E. Ainsworth. Validation of the COSMED K4 b2 portable metabolic system. Int. J. Sports Med. 22:280-284, 2001. 
12. Mo-Suwan, L., S. Pongprapai, C. Junjana, and A. Puetpaiboon. Effects of a controlled trial of a school-based exercise program on the obesity indexes of preschool children. Am. J. Clin. Nutr. 68:1006-1011, 1998.

13. Ogden, C.L., M.D. Carroll, L.R. Curtin, M.A. McDowell, C.J. Tabak, and K.M. Flegal. Prevalence of overweight and obesity in the United States, 1999-2004. JAMA. 295:1549-1555, 2006.

14. Row, P.J., J.M. Schuldheisz, and H. Van Der Mars. Measuring physical activity in physical education: Validation of the SOFIT direct observation instrument for use with first to eight grade students. Pediatr. Exerc. Sci. 9:136-149, 1997.

15. Sallis, J.F., T.L. McKenzie, J.E. Alcaraz, B. Kolody, N. Faucette, and M.F. Hovell. The effects of a 2-year physical education program (SPARK) on physical activity and fitness in elementary school students. Am. J. Public Health. 87:1328-1334, 1997.

16. Scruggs, P.W., S.K. Beveridge, P.A. Eisenman, D.L. Watson, B.P. Shultz, and L.B. Ransdell. Quantifying physical activity via pedometry in elementary physical education. Med. Sci. Sports Exerc. 35:1065-1071, 2003.

17. Stewart, J.A., D.A. Dennison, H.W. Kohl, and J.A. Doyle. Exercise level and energy expenditure in the TAKE 10 ! in-class physical activity program. J. Sch. Health. 74:397-400, 2004.

\section{Acknowledgments}

This work was supported by grant NIHDK061489 from the National Institute of Diabetes and Digestive and Kidney Disease, Bethesda MD, awarded to Dr. Joseph E. Donnelly. 
Copyright of Pediatric Exercise Science is the property of Human Kinetics Publishers. Inc. and its content may not be copied or emailed to multiple sites or posted to a listserv without the copyright holder's express written permission. However, users may print, download, or email articles for individual use. 